\title{
LOW-FREQUENCY WIGGLER RADIATION
}

\section{F. Méot, CEA, DSM/DAPNIA/SEA, 91191 Saclay, France*}

\section{Abstract}

Classical formalism for synchrotron radiation interference between short sources is applied to analytical formulation of wiggler radiation in the low frequency domain.

\section{INTRODUCTION}

Considering the lasting interest in synchrotron radiation (SR) for beam diagnostics [1], due also to raising specific demand such as infrared SR [2], a detailed insight in the interference phenomenon between separate radiating sources and its understanding remains of concern. The case of wiggler SR has already been addressed in terms of interference [3] and more recently implications of the low frequency hypothesis have been discussed [4]. We re-visit the subject with recently developed material [5] and derive by this means a detailed analytical formulation of wiggler SR in the low frequency domain.

\section{ANALYTICAL MATERIAL}

\subsection{Low frequency model}

In regular conditions of SR production the spectral angular energy density observed at large distance $r$ (assumed constant) is given by $\frac{\partial^{3} W}{\partial \omega \partial \phi \partial \psi}=2 \epsilon_{0} c\left|{ }^{r} \tilde{\vec{E}}(\phi, \psi, \omega)\right|^{2}$ (the $r$-independent quantity ${ }^{r} \tilde{\vec{E}}=r \tilde{\vec{E}}$ is introduced for simplicity) where $\tilde{\vec{E}}(\phi, \psi, \omega)$ is the Fourier transform of the radiated electric field $\vec{E}(\phi, \psi, t)$ (see Fig. 1) and $\omega$ is the observed frequency. In the low frequency domain one has

$$
\begin{aligned}
{ }^{r} \tilde{E}_{\sigma}(\phi, \psi, \omega) & =\frac{q \gamma}{(2 \pi)^{3 / 2} \epsilon_{0} c}\left(\frac{K-\gamma \phi}{1+(K-\gamma \phi)^{2}+\gamma^{2} \psi^{2}}\right. \\
& \left.+\frac{K+\gamma \phi}{1+(K+\gamma \phi)^{2}+\gamma^{2} \psi^{2}}\right) \\
{ }^{r} \tilde{E}_{\pi}(\phi, \psi, \omega) & =\frac{q \gamma}{(2 \pi)^{3 / 2} \epsilon_{0} c} \gamma \psi\left(\frac{1}{1+(K-\gamma \phi)^{2}+\gamma^{2} \psi^{2}}\right. \\
& \left.-\frac{1}{1+(K+\gamma \phi)^{2}+\gamma^{2} \psi^{2}}\right)
\end{aligned}
$$

where indices $\sigma$ and $\pi$ designate polarisation components respectively parallel to the bend plane and normal to $\overrightarrow{E_{\sigma}}$; angles $\phi$ in the bend plane and $\psi$ normal to it define the observation direction. Eqs. 1 holds over a few rms aperture

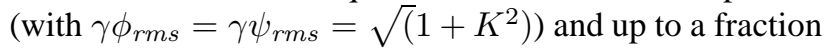
of $\omega_{\text {limit }}$ as defined by

$$
\omega_{\text {limit }}=\omega_{c} /\left(3 K\left(1+K^{2}\right)\right)=\gamma^{2} c /\left(L\left(1+K^{2}\right)\right)
$$

in which $\omega_{c}=3 \gamma^{3} c / 2 \rho$ is the critical frequency of the standard formalism, $\gamma$ is the Lorentz relativistic factor, $\rho$ is

\footnotetext{
*Email : fmeot@cea.fr
}
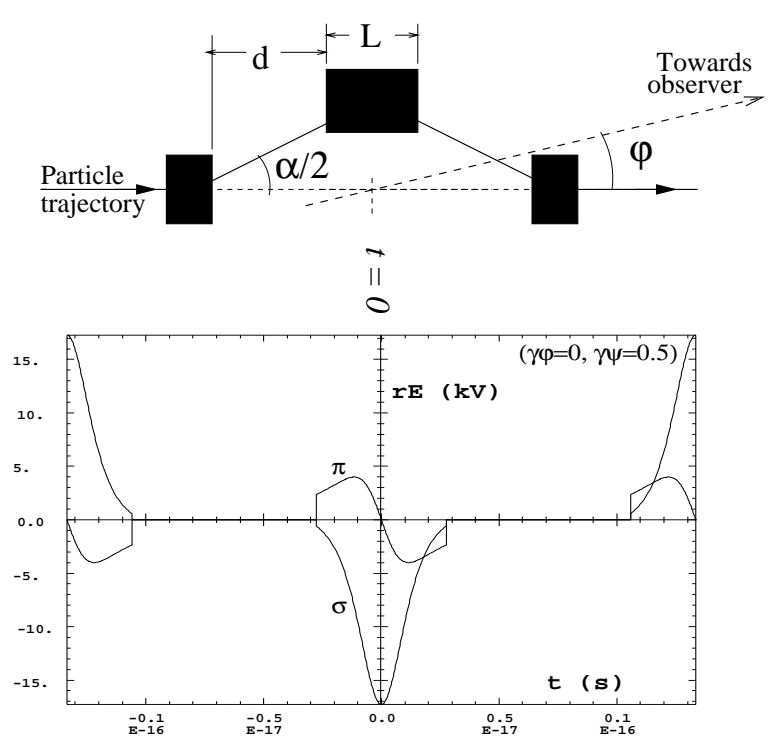

Figure 1: $U p$ : A three-dipole wiggler. Definition of observation angle $\varphi$ in the bend plane. Down: Typical shape of the electric field impulse ${ }^{3 r} E_{\sigma, \pi}(\varphi, \psi, t)$ at the observer.

the curvature radius, $\alpha$ is the particle total deviation, $L=$ $\rho \alpha$ is the trajectory length in the magnetic field, and $K=$ $\alpha \gamma / 2$ is the deflection parameter.

\subsection{Interference}

As one knows the underlying physics in SR interference is in time coherence resulting from the geometrical arrangement of sources, which entails space and frequency modulation of the radiated signal. A series of $N$ sources radiate electric field of the form

$$
{ }^{N} E_{\sigma, \pi}(\phi, \psi, t)=\sum_{i=1}^{N} \delta\left(t+T_{i}\right) * E_{i \sigma, \pi}(\phi, \psi, t)
$$

where $E_{i_{\sigma, \pi}}(\phi, \psi, t)$ describes the impulse from magnet $i$, $T_{i}$ is the emission time of signal $i, \delta$ is the Dirac distribution and $*$ denotes the convolution product. The Fourier transform gives the interferential amplitude density

$$
{ }^{N} \tilde{E}_{\sigma, \pi}(\phi, \psi, \omega)=\sum_{i=1}^{N} e^{i \omega T_{i}} \tilde{E}_{i_{\sigma, \pi}}(\phi, \psi, \omega)
$$

whose modulus square provides the energy density

$$
\begin{aligned}
\frac{\partial^{3 N} W_{\sigma, \pi}}{\partial \omega \partial \phi \partial \psi} & =2 \epsilon_{0} c\left(\left(\sum_{i=1}^{N}{ }^{r} \tilde{E}_{i_{\sigma, \pi}} \cos \left(\omega T_{i}\right)\right)^{2}\right. \\
& \left.+\quad\left(\sum_{i=1}^{N}{ }^{r} \tilde{E}_{i \sigma, \pi} \sin \left(\omega T_{i}\right)\right)^{2}\right)
\end{aligned}
$$


Times $T_{i}$ are obtained from the geometry of the magnet assembly by combining the duration $\Delta T=\frac{L}{2 \gamma^{2} c}(1+$ $\left.K^{2} / 3+\gamma^{2}\left(\phi^{2}+\psi^{2}\right)\right)$ of the impulse issued from a magnet, with the time of flight (in observer time) $\Delta T_{d}=$ $\frac{d}{2 \gamma^{2} c}\left(1+\gamma^{2}\left(\phi^{2}+\psi^{2}\right)\right)$ between magnets distant $d$.

Note that Eqs. 4, 5 involve the exact Fourier transform of $\delta\left(t+T_{i}\right)$ hence possible low frequency approximation validity domain depends only on the characteristics ( $L$ and $K)$ of SR sources, not on their distance $d$.
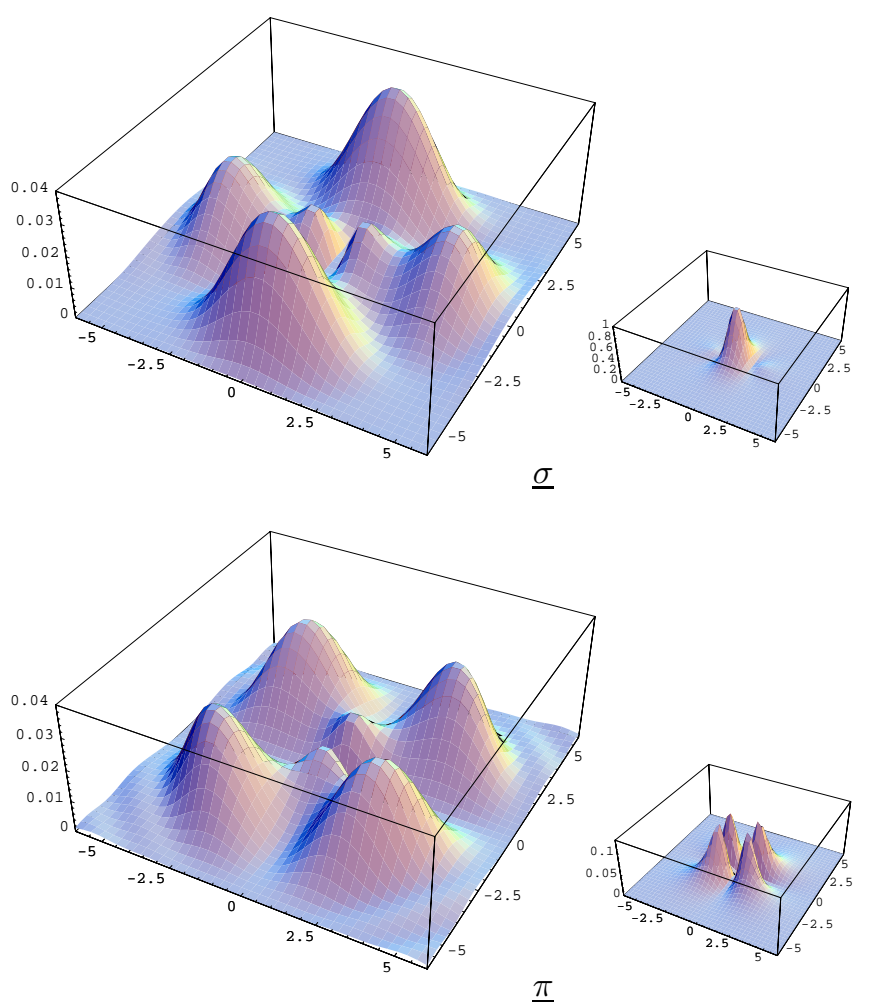

Figure 2: Low frequency energy density from a three-dipole wiggler (Eq. 6 for $2.5 \mathrm{GeV}$ electron, at $\omega=1.910^{15} \mathrm{rad} / \mathrm{s}$ $\left.\lambda=10^{-6} \mathrm{~m}\right)$. The small boxes show the energy density from the central $(2 / \gamma)$ dipole alone ; comparison reveals a damping of about $0.04 / 1$ due to the wiggler interference. (g.phi and g.psi stand for coordinates $\gamma \phi$ and $\gamma \psi$.)

\section{SR FROM A 3-DIPOLE WIGGLER}

For simplicity a single-bump wiggler based on $(1 / \gamma)$ - and $(2 / \gamma)$-deviation magnets ${ }^{1}$ is considered (Fig. 1$)$. However what follows can be extended to arbitrary $N$, and as well to low frequency undulator radiation ( $N$ large and $K<1$ ).

The low frequency limit simplifies into $\omega_{\text {limit }} \approx \omega_{c} / 6=$ $\gamma^{2} c / 2 L$ (Eq. 2 with $K=1$ ) leading for instance to validity range $\omega<410^{16} \mathrm{rad} / \mathrm{s}\left(\lambda>4010^{-9} \mathrm{~m}\right)$ for a $2.5 \mathrm{GeV}$ electron traversing a, e.g., $670 \mathrm{kG}, L=510^{-2} \mathrm{~m}$ long dipole. Fig. 1 shows the typical shape of electric field impulse series so generated (Eq. 3 with $N=3$ ), the total

$$
\text { SR. }
$$

\footnotetext{
${ }^{1}$ The latter has the merit of producing highest brightness low frequency
}

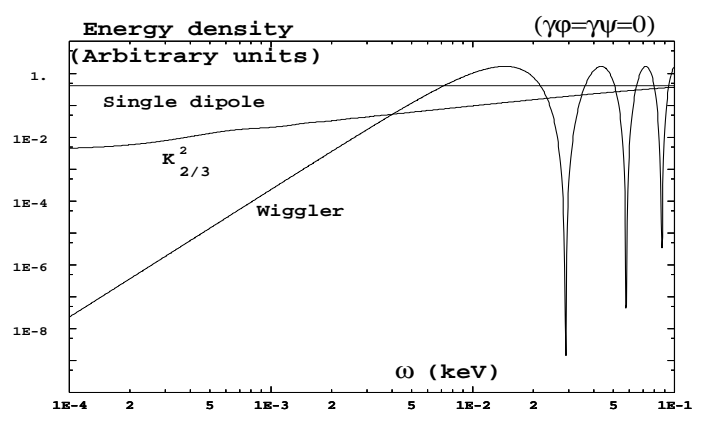

Figure 3: Forward spectrum of wiggler $\sigma$ component (Eq. 6), and for comparison, spectra due to the central $(2 / \gamma)$-deviation dipole alone ( $K=1$ in Eq. 1$)$, and due to body SR from a strong dipole (regular $K_{2 / 3}^{2}(\omega)$ shape).

duration of which is $\left.\Delta T\right|_{\frac{L}{2}, \frac{K}{2}, \phi=\varphi-\frac{K}{2 \gamma}}+\left.\Delta T_{d}\right|_{\phi=\varphi-\frac{K}{\gamma}}$ $+\left.\Delta T\right|_{\phi=\varphi}+\left.\Delta T_{d}\right|_{\phi=\varphi+\frac{K}{\gamma}}+\left.\Delta T\right|_{\frac{L}{2}, \frac{K}{2}, \phi=\varphi+\frac{K}{2 \gamma}}$. Taking the origin at the centre of the central dipole, times in Eq. 3 write, $T_{2}=0$ and

$$
\begin{gathered}
-T_{1}=\left.\Delta T\right|_{\frac{L}{2}, \frac{K}{2}, \phi=\varphi-\frac{K}{2 \gamma}}+\left.\Delta T_{d}\right|_{\phi=\varphi-\frac{K}{\gamma}}+\left.\frac{1}{2} \Delta T\right|_{\phi=\varphi} \\
+T_{3}=\left.\frac{1}{2} \Delta T\right|_{\phi=\varphi}+\left.\Delta T_{d}\right|_{\phi=\varphi+\frac{K}{\gamma}}+\left.\Delta T\right|_{\frac{L}{2}, \frac{K}{2}, \phi=\varphi+\frac{K}{2 \gamma}}
\end{gathered}
$$

Fig. 2 shows the resulting interferencial patterns which express as

$$
\begin{gathered}
\frac{\partial^{33} W_{\sigma, \pi}}{\partial \omega \partial \varphi \partial \psi}=2 \epsilon_{0} c \\
\left(\left(\left.{ }^{r} \tilde{E}_{\sigma, \pi}\right|_{\frac{K}{2}, \phi=\varphi-\frac{K}{2 \gamma}} \cos \left(\omega T_{1}\right)+\left.{ }^{r} \tilde{E}_{\sigma, \pi}\right|_{-K, \phi=\varphi}\right.\right. \\
\left.\left. \pm\left.{ }^{r} \tilde{E}_{\sigma, \pi}\right|_{\frac{K}{2}, \phi=\varphi+\frac{K}{2 \gamma}} \cos \left(\omega T_{3}\right)\right)^{2}+(S T)^{2}\right)
\end{gathered}
$$

where $(S T)$ designates the complementary sin term. Fig. 3 compares the radiation spectrum from the wiggler to that of a single $K=1$ dipole and to classical body SR from a $K \gg 1$ dipole.

\section{REFERENCES}

[1] A. Hofmann, Diagnostics with SR, Proc. Cern Acc. School "Synchrotron Radiation and Free-Electron Lasers", Grenoble (F), 22-28 April 1996.

[2] Workshop on infrared SR, $1^{\text {st }}$ meeting : the source, J.-L. Laclare, M.-P. Level and P. Roy organisers, Gif-sur-Yvette, France, 8-9 Dec. 1997.

[3] K.-J. Kim, Characteristics of SR, AIP Conf. Proc. 184, US Part. Acc. School, Fermilab, 1987 ; R. P. Walker, Interference effects in undulator and wiggler radiation sources, Internal Report Sincrotrone Trieste, 1993.

[4] R. A. Bosch, Long-wavelength radiation along the straightsection axis in anelectron storage ring, NIM A, vol. 386 (1997) 525-530.

[5] F. Méot, A theory of low frequency far-field synchrotron radiation, report DSM/DAPNIA/SEA-98-05, CEA/Saclay (1998) ; F. Méot, N. Ponthieu, On low frequency far-field interference between short SR sources, report DSM/DAPNIA/SEA-98-53, CEA/Saclay (1998). 
Proceedings of the 1999 Particle Accelerator Conference, New York, 1999 